\title{
Internet das coisas, Games e Data Science a serviço da conscientização e preservação da Caatinga
}

\author{
Geraldo Cruz-Júnior ${ }^{1,4}$, Rafaella Nascimento ${ }^{3}$, Gilberto Cysneiros Filho1, Ednilza \\ Santos $^{2}$, Gabriel Alves ${ }^{1}$ \\ ${ }^{1}$ Departamento de Estatística e Informática - Universidade Federal Rural de \\ Pernambuco \\ Recife - PE - Brasil \\ ${ }^{2}$ Departamento de Ciências Biológicas - Universidade Federal Rural de Pernambuco \\ Recife - PE - Brasil \\ ${ }^{3}$ Escola Politécnica - Universidade de Pernambuco \\ Recife - PE - Brasil \\ ${ }^{4}$ Instituto SENAI de Inovação para Tecnologias da Informação e Comunicação, SENAI, \\ Recife - PE - Brasil \\ \{geraldoj8, rafaellalsn, g.cysneiros, ednilzamaranhao, \\ gaaj1980\}@gmail.com
}

\begin{abstract}
This article presents to the community a mobile and web project that joins educational games, internet of things and data science to aid in the dissemination, education and preservation of the caatinga ecosystem. The tool enables an integration between the population and researchers, where both parties can use it in an ongoing process of learning, providing information, expanding the research and dissemination of the caatinga. The tool was evaluated by researchers, members of environmental non-governmental organizations (NGOs) and students. The use of the tool made it possible to obtain data that show its relevance to the region's knowledge and research.
\end{abstract}

Resumo. Este artigo apresenta para a comunidade um projeto mobile e web que une jogos educativos, internet das coisas e data science para auxílio na divulgação, educação e preservação do ecossistema da caatinga. A ferramenta possibilita uma integração entre população e pesquisadores, onde ambas as partes podem utilizá-la em um processo contínuo de aprendizado, fornecendo informações, ampliando o desenvolvimento de pesquisas e difusão da caatinga. A ferramenta foi avaliada por pesquisadores, membros de Organizações Não Governamentais (ONG) de preservação ambiental $e$ estudantes. A utilização da ferramenta possibilitou a obtenção de dados que mostram sua relevância para o conhecimento da região e pesquisas.

\section{Introdução}

A caatinga é o único bioma exclusivamente brasileiro, ocupando uma área de cerca de $850.000 \mathrm{~km}^{2}$, que consiste em $11 \%$ do território nacional e aproximadamente $70 \%$ da região nordestina [Brasil 2017; Drumond et. al 2000]. Com mais de 300 espécies 
vegetais exclusivas desta região e agrupando um total de 1.225 espécies de animais vertebrados catalogados, dentre eles alguns ameaçados de extinção como a arara-azul. No entanto, ainda não existe uma lista completa para as espécies existentes na caatinga, encontradas nas suas mais diferentes situações edafoclimáticas (Agreste, Sertão, Cariri, Seridó, Carrasco, entre outros) [Drumond et. al 2000]. Descrições de novas espécies vêm sendo registradas com frequência, indicando um conhecimento botânico e zoológico bastante precário deste ecossistema, que é considerado o menos conhecido e estudado do Brasil.

Este patrimônio ecológico está ameaçado, pois cerca de $70 \%$ da caatinga já se encontra alterada pelo homem e somente $0,28 \%$ de sua área encontra-se protegida em unidades de conservação. De acordo com o Ministério do Meio Ambiente (MMA), resta cerca de 54\% da cobertura vegetal original [Brasil 2017]. Para reverter este processo, estudos da flora e fauna da caatinga são necessários. Neste sentido, surgem projetos para a preservação da caatinga que têm como principal objetivo sensibilizar as pessoas a cuidar da natureza, fazendo pesquisas, disseminando o conhecimento e mostrando importância da preservação deste bioma. Atualmente, há vários projetos de parceria entre o MMA e os estados, a fim desta conscientização [Brasil 2017].

Com a visão de contribuir com os esforços das iniciativas governamentais e privadas de preservação ambiental, este estudo descreve um software desenvolvido a partir de uma parceria entre o Departamento de Biologia e o Departamento de Estatística e Informática da Universidade Federal Rural de Pernambuco. A ideia da ferramenta é possibilitar a colaboração entre população e pesquisadores da área, fazendo com que ambas as partes possam utilizá-la para fins de conhecimento, fornecimento de informações e contribuição para pesquisas sobre a caatinga.

Para isto, são utilizados os conceitos de gamefication, unidos as principais necessidades apontadas por organizações não governamentais (ONGs) da caatinga para se desenvolver games educativos de diferentes gêneros com o intuito de conscientizar e educar a população em relação a este importante ecossistema. As necessidades das ONGs foram obtidas durante o processo de pesquisa por meio de entrevistas. Com esta funcionalidade é possível que sejam levantadas estatísticas e estimado o conhecimento que as pessoas da região possuem sobre este bioma. $\mathrm{O}$ aplicativo também possibilita que os usuários denunciem possíveis casos de crime ambiental anexando depoimentos e fotos a serem enviadas para as autoridades responsáveis. No âmbito das pesquisas, a ferramenta ainda propicia a criação de um banco de dados de categorização de espécies para os pesquisadores de ONGs da região, servindo como agente informante e catálogo de prováveis novas espécies identificadas. Os usuários podem tirar fotos e documentar algum animal ou vegetação que achem interessante.

A internet das coisas é utilizada em união com a data science na coleta e análise dos dados oriundos dos dispositivos dos usuários, do banco de dados fornecidos pelo Instituto Brasileiro de Geografia e Estatística (IBGE) e de ONGs da caatinga. O resultado do cruzamento destes dados é ilustrado em gráficos e tabelas informativas. Todas as funcionalidades disponibilizadas pelo sistema podem ser utilizadas tanto via web, como via aplicativo Android, IOS e Windows Phone. O Sistema desenvolvido e apresentado neste trabalho atende as principais necessidades apontadas por ONGs da região, ajudando a enriquecer o trabalho realizado por grupos de pesquisa na caatinga. 
VI Congresso Brasileiro de Informática na Educação (CBIE 2017)

Anais do XXVIII Simpósio Brasileiro de Informática na Educação (SBIE 2017)

Como metodologia, é utilizado o Toolkit Human-Centered Design (HCD) somado a uma abordagem qualitativa de caráter exploratório-descritivo.

\section{Trabalhos relacionados}

O trabalho de Siqueira et. al (2012) descreve um sistema para manipulação e gerenciamento eletrônico de coleções botânicas. O software descrito foi desenvolvido com objetivo de auxiliar herbários brasileiros no resgate e manutenção segura de registros de espécies de plantas provenientes de diversos ecossistemas nacionais. A ferramenta possui funcionalidades para cadastro, consultas, atualizações, estatísticas, relatórios, geração de etiquetas, geoprocessamento e banco de imagens.

Segundo Panosso et. al (2015), há muitas maneiras de arranjar contingências para promover a aprendizagem, sendo os jogos educativos uma delas. Para Dondi e Moretti (2007), os jogos educativos são definidos como aqueles que possuem um objetivo didático explícito e podem ser adotados ou adaptados para melhorar, apoiar ou promover os processos de aprendizagem em um contexto de aprendizagem formal ou informal. Martins et. al (2013) expõe que a presente degradação da biodiversidade necessita de estratégias para a conscientização de preservação ambiental, sendo os jogos uma das abordagens aplicadas. Sendo assim, é realizado um trabalho onde procurou-se analisar jogos sobre biodiversidade, em relação as suas potencialidades e limitações.

Além dos jogos educacionais, outras ferramentas tecnológicas podem ajudar na conscientização ambiental. Rocha et. al (2015) apresentam o desenvolvimento de um aplicativo que efetua leitura de Códigos QR provenientes de identificações botânicas das espécies arbóreas da Trilha de Educação Ambiental inserida na Reserva Biológica de Sooretama. O aplicativo oferece a oportunidade de trabalhar a educação ambiental de forma mais flexível, apresentando os conteúdos de uma maneira mais atraente e lúdica

Tendo em vista os trabalhos expostos, o desenvolvimento da ferramenta proposta nesse artigo mostra-se de importante ganho a comunidade científica e a população de forma geral. As necessidades são abrangidas pois a ferramenta une diversas funcionalidades de forma centralizada podendo ajudar ao bioma da caatinga: os games desenvolvidos como forma de educação ambiental, a ferramenta de catalogação de espécies para pesquisadores, o envio de ameaças encontradas nas áreas de preservação, recursos como geração de relatórios, gráficos e estatísticas.

\section{Metodologia}

De acordo com IDEO (2014), o ato de projetar soluções inovadoras e relevantes, que atendam às necessidades, desejos e comportamentos das pessoas, começa com o entendimento de suas necessidades, expectativas e aspirações para o futuro. Para o desenvolvimento de um projeto desta natureza, o qual é voltado para diferentes perfis de usuários e cujo objetivo é o auxílio na compreensão e combate a uma problemática complexa e que envolve várias variáveis, não basta apenas o interesse e a vontade de tentar ajudar e colaborar com a preservação da caatinga, é preciso, sobretudo, observar a problemática de perto, ouvir especialistas e compreender as reais necessidades apontadas pelos stakeholders envolvidos.

Desta forma, utilizou-se a combinação de duas metodologias para o desenvolvimento desta pesquisa. A primeira, é o Toolkit Human Centered Design 
VI Congresso Brasileiro de Informática na Educação (CBIE 2017)

Anais do XXVIII Simpósio Brasileiro de Informática na Educação (SBIE 2017)

(HCD), que inicia com a definição de um desafio estratégico específico e prossegue por três fases principais, são elas: Ouvir, Criar e Implementar. O foco do Toolkit HCD é construir soluções colaborativamente, através do somatório de diferentes perspectivas e visões [IDEO 2014]. Somado a esta metodologia utilizou-se uma abordagem qualitativa de caráter exploratório-descritivo, a partir das análises das principais observações feitas relativas a caatinga. $\mathrm{O}$ estudo exploratório é definido como uma pesquisa que tem por objetivo proporcionar maior familiaridade com um problema, a fim de torná-lo mais explícito, principalmente ao tratar-se de um tema pouco investigado [Luciano et. al 2007].

Visando a ordenação do processo metodológico da pesquisa, aqui são apresentadas três subseções, cada uma representando uma fase do Tookit HCD, passando pela Fase Ouvir, Fase Criar e por fim, a Fase Implementar.

\subsection{Fase Ouvir}

O objetivo desta primeira fase é determinar quem são os especialistas que devem ser abordados para o projeto, também espera-se ganhar empatia com os possíveis usuários e coletar histórias de interesse [IDEO 2014]. Visando orientar a elaboração de perguntas que são feitas na pesquisa de campo com o público-alvo do projeto, precisa-se definir um desafio estratégico. O desafio proposto para este trabalho consiste em responder a seguinte pergunta: "Como fazer com que as pessoas tenham conhecimento especializado sobre o ecossistema da caatinga de forma lúdica e simplificada?".

A equipe do projeto é formada por integrantes da Ciência da Computação e da Biologia, mais especificamente, especialistas no desenvolvimento de aplicações mobile/web e especialistas do ecossistema da caatinga, respectivamente. Desta forma, conciliando o conhecimento da equipe, chegou-se a hipótese inicial de desenvolver uma solução de software que pudesse transmitir informações referentes a caatinga para $o$ usuário final de uma forma não trivial, mas sim divertida e simplificada. Após elencar os conhecimentos preexistentes da equipe, foram levantadas perguntas com o objetivo de conhecer profundamente as necessidades do público-alvo.

Dentre as perguntas elaboradas, tem-se: (I) O que fazem, pensam ou sentem os membros da comunidade, pesquisadores e cidadãos da região, em relação ao conhecimento da Caatinga? (II) $\mathrm{O}$ que as pessoas acham das ofertas de aplicativos disponíveis neste contexto? (III) Quais os principais desafios para implementar as ideias do projeto? (V) Onde estão as maiores necessidades de pesquisa? (VI) Se o aplicativo existisse, como ele seria? (VII) Porque você [não] acharia interessante conhecer mais sobre a Caatinga? (VIII) Conte-nos como você faz para obter informações e realizar seus trabalhos relacionados a Caatinga? (IX) Por que você [não] utiliza um sistema para obter estas informações? (X) Fale-nos como é utilizar o seu recurso para conhecimento da Caatinga? (XI) Conte-nos como você [não] se diverte ao utilizar esse recurso e o quanto ele é simples? (XII) Você possui smartphone ou tablet?

Como público alvo foi selecionado um grupo de Caatingueiros, uma turma de estudantes do ensino fundamental e pesquisadores de ONGs da região e universidades. Foram realizadas entrevistas individuais e em grupo para se entender as necessidades de cada grupo. As entrevistas individuais foram realizadas por preenchimento de um formulário online. Todas as entrevistas em grupo ocorreram nos ambientes da UFRPE e na Escola Francisco Simões da Costa, localizada no município de Igarassu, 
VI Congresso Brasileiro de Informática na Educação (CBIE 2017)

Anais do XXVIII Simpósio Brasileiro de Informática na Educação (SBIE 2017)

Pernambuco. Algumas entrevistas com pessoas consideradas experts e de conhecimento relevante para a pesquisa foram realizadas por trocas de e-mail, Skype e ligações.

\subsection{Fase Criar}

O objetivo desta segunda fase é entender os dados, identificar padrões, definir oportunidades e criar soluções. O Toolkit HCD alerta que para transformar pesquisas em soluções é preciso filtrar e selecionar informações, traduzindo insights em oportunidades. Assim, adota-se um ponto de vista generativo para criar diversas soluções em brainstorms e rapidamente converter algumas delas em protótipos [IDEO 2014]. Inicialmente houve a interpretação das histórias ouvidas durante a pesquisa de campo, para transformá-las em dados que são utilizados para inspirar a criação de oportunidades, ideias e soluções. Neste trabalho utilizou-se a técnica de extração de insights para identificação de padrões e informações relevantes nos dados extraídos das entrevistas. Com todos os principais insights selecionados, a equipe do projeto realizou um brainstorm de possíveis soluções para os problemas que foram destacados.

Com o resultado das entrevistas, a extração de insights e o brainstorm, pode-se chegar em uma solução mais completa e refinada que atendesse as necessidades dos diferentes públicos dentro de uma mesma plataforma. $\mathrm{O}$ que inicialmente seria apenas jogos educativos, ganhou o acréscimo de módulos de denúncia de crimes ambientais, um catálogo de espécies vegetais e animais da região e uma plataforma para centralizar e compartilhar dados, informações e notícias referentes a caatinga. Neste momento se definiu o escopo principal do projeto que foi intitulado de Caatinga Digital.

O projeto Caatinga Digital conta com os módulos: (I) Jogos: São 4 jogos clássicos, Quiz, Forca, Memória e Quiz por Imagens, adaptados para instruir e informar sobre a Caatinga enquanto se joga; (II) Estatísticas: Mapas, gráficos, tabelas e relatórios referentes a dados do IBGE, ONGs e pesquisadores; (III) Categorização: Catálogo de espécies animais e vegetais da região, que permite o cadastro de novas espécies; (IV) Denúncias: Espaço para que a população possa denunciar crimes ambientes que estejam ocorrendo; (V) Notícias: Feed com canais de notícias ambientais.

A partir da definição dos módulos do projeto desenvolveu-se o protótipo da plataforma. O protótipo usa técnicas de design com tendências do Flat Design, que é um padrão de estética que visa a uma interface simples, limpa e direta [Pratas 2014]. Neste projeto ainda se fez uso dos critérios de usabilidade de Jakob Nielsen, que são: facilidade de aprendizado; facilidade de recordação; eficácia; eficiência; segurança no uso e satisfação do usuário [Nielsen e Budiu 2013]. Estes foram utilizados para certificar que a plataforma fosse simples e usual para diferentes públicos.

Com o protótipo finalizado, o público-alvo foi consultado para a coleta de feedbacks deste. Nesta fase, se busca a obtenção de opiniões qualitativas e quantitativas, a fim de identificar se o protótipo foi aceito, bem como capturar novas ideias e possíveis melhorias pois, quanto maior a usabilidade de uma interface, mais fácil será de utilizála, por isso são essenciais no desenvolvimento de software [Ferreira et. al 2014].

A equipe de desenvolvimento do Caatinga Digital procurou responder as seguintes questões após os feedbacks: (I) As soluções propostas proporcionam um conhecimento sobre a Caatinga e facilitam o seu trabalho? (II) As soluções são atrativas? (III) Você se divertiria aprendendo através de jogos? (IV) As funcionalidades 
existentes são suficientes? Você sugeriria mais alguma? (V) A obtenção de informações sobre a Caatinga está de uma forma facilitada? (VI) As soluções facilitariam as suas vidas, quanto ao conhecimento da região e obtenção de dados? (VII) O que está ruim? (VIII) O que você adicionaria?.

Para a realização dos testes do protótipo a equipe disponibilizou o arquivo instalável do projeto, juntamente com um tutorial para os usuários que possuíam dispositivos Android. Para os demais, foi repassado o acesso da versão web do protótipo. A equipe criou um ambiente sem interferências, onde os usuários puderam testar e criticar as funcionalidades livremente. Parte da obtenção dos feedbacks foi feita de forma remota, onde os usuários enviaram os seus feedbacks por e-mail, e outra parte foi obtida de forma presencial.

\subsection{Fase Implementar}

Após o desenvolvimento e coleta de feedbacks do protótipo, a equipe iniciou a fase de implementação do aplicativo, a fim de torná-lo factível. Os objetivos desta fase são: identificar capacidades e tecnologias necessárias, criar um modelo financeiro sustentável; desenvolver a sequência de projetos de inovação; criar pilotos e medir impactos [IDEO 2014]. Desta forma, tem-se como proposta de valor para os usuários finais a entrega de conhecimentos sobre a caatinga de forma facilitada e lúdica, através de jogos e diferentes módulos, livres de custos, ou seja, grátis nas lojas de aplicativos para smartphones e via web, que são os canais de distribuição mais acessíveis para o público entrevistado. Este modelo é possível pois o projeto é realizado em âmbito de pesquisas e tem apoio de instituições como a CNPQ e ONGs.

As capacidades requeridas para a realização do projeto são tanto os conhecimentos técnicos das ferramentas, onde foi utilizado o framework Angular e um servidor Apache, quanto as linguagens de programação Javascript e PHP, unidas ao contexto de interface com o CSS3 e o HTML5. Por fim, o acesso aos dados e a persistência se deu por uso do gerenciador MySQL. Estas tecnologias juntas garantem que o projeto seja executado em diferentes plataformas ou dispositivos. A promoção do data science nos dados capturados pela aplicação é possível graças ao PHP Statistic e ao Jstat. A internet das coisas acontece com a captura de informações dos dispositivos dos usuários, que quando conectados a internets sincronizam seus dados com o servidor da aplicação. Todo o projeto foi versionado em Git e seus códigos estão disponíveis de forma open source no GitHub.

Também é fundamental que os dados e informações fornecidas na aplicação tenham integridade e confiabilidade confirmadas. Para isto, o sistema faz uso de dados do IBGE, de ONGs da região, de grupos de pesquisa e de canais de notícia confiáveis, que alimentam os jogos e módulos do Caatinga Digital.

Os dados dos resultados dos jogos, envios de denúncia e adição de espécies ao categorizador, por exemplo, são capturados dos dispositivos dos usuários e sincronizados com o web service da aplicação. Estes, juntos com os dados capturados do IBGE e de ONGs são disponibilizados via API tanto para outros desenvolvedores que desejem utilizá-los, como para a própria aplicação que faz uso do data science, com algoritmos de identificação de outliers, correlações e medidas de posição e dispersão, para analisar os dados e exibir informações via tabelas, gráficos e mapas. Isso mostra o poder da internet das coisas, pois qualquer dispositivo com conexão à internet pode 
VI Congresso Brasileiro de Informática na Educação (CBIE 2017)

Anais do XXVIII Simpósio Brasileiro de Informática na Educação (SBIE 2017)

utilizar a aplicação e a partir dela ingressar numa rede de colaboração e troca de informações sobre a caatinga.

Como planejando futuro estabeleceu-se dois marcos: a fase em que se encontra o projeto, e para onde o mesmo deverá avançar. Em suma, a aplicação está em sua versão atual em fase de testes, para análise de aceitação dos usuários. Um aspecto bastante positivo do projeto é que mesmo em sua fase de avaliação, já se recebe muitos feedbacks positivos do público alvo. A aplicação já está em pleno uso para alguns pesquisadores, estes já demostram grande aceitação desta e ressaltam o quanto ela é útil em seu cotidiano. Espera-se frente aos feedbacks recebidos da versão atual, preparar e disponibilizar o projeto em canais públicos, como a Play Store, a App Store e via web.

Visto que é primordial estar preparado para possíveis mudanças, melhorias e aprendizados frente a solução que foi proposta inicialmente, foi desenhado um plano de aprendizagem. O Caatinga Digital conta com ferramentas para a realização de análises qualitativas e quantitativas de dados referentes à quantidade de usuários, comentários, opiniões, dispositivos utilizados, países que usam a aplicação, dentre outras informações oriundas do gerenciador de aplicativos das lojas que hospedam o projeto.

\section{Resultados}

A avaliação de um jogo educacional é imprescindível para constatar se ele atende aos requisitos pedagógicos e de usabilidade necessários para motivação e engajamento dos usuários [An et. al 2013]. Na busca por respostas com base nas entrevistas, feedbacks e avaliações dos usuários, e visando a continuamente aperfeiçoar o projeto, a equipe inferiu sobre os dados obtidos nas três fases do projeto: Ouvir, Criar e Implementar. Aqui são apresentadas três seções, cada uma representando os resultados destas fases.

\subsection{Fase Ouvir}

Tendo em mente o desafio estratégico proposto para o projeto, avaliou-se qualitativamente e quantitativamente os resultados das entrevistas. Foram 52 pessoas entrevistadas, de diferentes idades, gêneros e necessidades com o aplicativo. Foram 42 alunos do ensino fundamental, 8 pesquisadores e 2 caatingueiros. Quanto a análise dos resultados da entrevista enveredou-se por uma abordagem mais qualitativa do que quantitativa, observando a opinião dos entrevistados quanto ao que foi questionado. Cerca de $88 \%$ das entrevistas ocorreram de forma presencial e $12 \%$ por vias online.

Em relação ao conhecimento sobre a caatinga, foi visível o desconhecimento deste bioma por parte dos alunos do ensino fundamental, todos os 52 entrevistados disseram que não conhecem ferramenta de software que dê suporte ao aprendizado sobre a região. Quanto aos desafios, necessidades de pesquisa e em como seria o aplicativo, os usuários especialistas da área tiveram respostas mais construtivas, apontando para possíveis funcionalidades até então não vistas pela equipe, como um categorizador de espécies animais e vegetais com a possibilidade de tirar fotos, o que vai ajudar no trabalho de pesquisadores em campo. Também foi sugerida uma funcionalidade para poder denunciar crimes ambientais e outra para disponibilizar informações do IBGE e do MMA na aplicação.

Por fim, também foi possível perceber que todos os entrevistados afirmaram possuir smartphone ou tablet. Em relação ao aprendizado sobre a caatinga prevaleceu a 
resposta que seria interessante uma solução mais interativa e se possível "gameficada" para aprender o conteúdo. Todos os entrevistados mostraram interesse sobre a provocação levantada e sugestões para diferentes funcionalidades que considerariam importantes. Os resultados destas observações serviram como base para a inclusão de novos módulos ao projeto frente ao que havia sido idealizado inicialmente.

\subsection{Fase Criar}

Com os resultados da Fase Ouvir já concretizados e analisados, foi observada a necessidade de se adicionar novos módulos ao projeto. Por fim, chegou-se ao Caatinga Digital, uma solução que conta com módulos de: Jogos Educativos, Estatísticas, Categorização de Flora e Fauna, Denúncia de Crimes Ambientais e Notícias. O protótipo do sistema foi implementado utilizando características do flat design e as heurísticas de usabilidade de Jakob Nielsen, para garantir que além das funcionalidades estarem de acordo com o que foi apontado pelos entrevistados, a solução também fosse elegante e simples de se utilizar.

Visando a obtenção de opiniões qualitativas e quantitativas para identificar a aceitação do protótipo, bem como capturar novas ideias e possíveis melhorias, elaborou-se uma novada rodada de entrevistas com o público-alvo, buscando a coleta de feedbacks do protótipo sugerido. Nesta fase foram 56 entrevistados, 42 alunos do ensino fundamental, 12 pesquisadores e 2 caatingueiros. $89 \%$ testaram o protótipo pelo Android e 11\% via web.

Quanto a análise das respostas da entrevista realizada nesta fase, foi observado que a maioria dos entrevistados responderam que a solução proposta proporciona facilidade na obtenção de conhecimentos sobre a Caatinga. Os pesquisadores ressaltaram o quanto a ferramenta pode vir a ajudar algumas de suas atividades em campo, salas de aula e pesquisas. A interface foi elogiada e os jogos foram apontados como atrativos para aprendizagem por alunos e professores. Analisando os feedbacks recebidos percebe-se que cerca de $93 \%$ foram positivos, exaltando as funcionalidades e a utilidade desta no dia-a-dia, cerca de $7 \%$ foram negativos, correspondem a 4 pessoas. O ponto negativo apontado foi relativo a um bug existente no protótipo que foi corrigido na versão seguinte da aplicação.

\subsection{Fase Implementar}

Diante das análises dos resultados de todas as fases neste percurso metodológico, foi proposta a primeira versão do Caatinga Digital (Figura 1), um projeto multiplataforma, de interface simplificada e usual, que une jogos, informações e serviços focados em promover o ensino da caatinga com diversão, facilidade de pesquisas e a preservação deste ecossistema. Por ser multiplataforma e responsivo garante um alcance maior dos seus benefícios para a população, sendo executado em diferentes sistemas operacionais. 

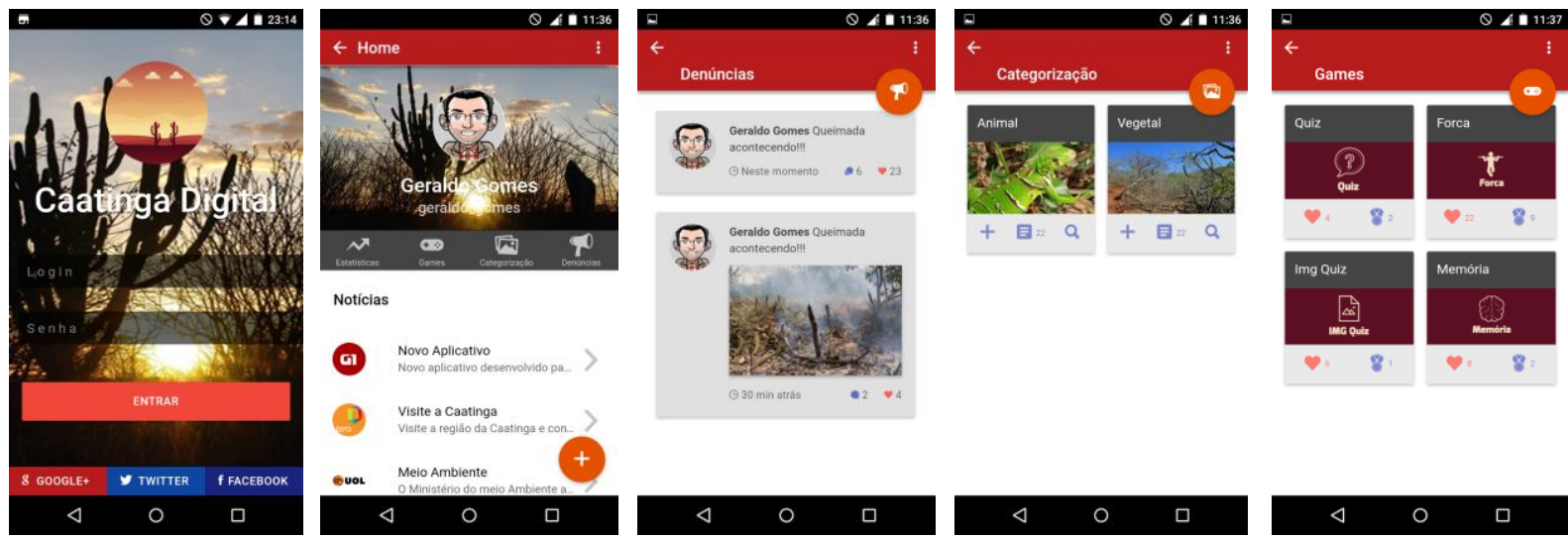

Figura 1. Telas do aplicativo Caatinga Digital

Todos os cinco módulos (Jogos Educativos, Estatísticas, Categorização de Flora e Fauna, Denúncia de Crimes Ambientais e Notícias) foram desenvolvidos frente as necessidades apontadas pelo público alvo, foram desenvolvidos em paralelo com os entrevistados, validando as funcionalidades e interfaces. Houve uma preocupação quanto aos jogos, para que estes fossem simples de se jogar e que realmente houvesse aprendizado enquanto se joga. Por isso, estes seguem modelos de games difundidos e conhecidos pelo público. Quanto a aprendizagem, os jogos foram desenvolvidos em paralelo com as necessidades e requisitos apontados pelos professores para que houvesse uma aprendizagem simplificada e divertida sobre a caatinga.

O projeto possui uma página na internet, que pode ser acessada em: http://mobileufrpe.com.br/caatingadigital/, esta contém mais informações sobre o desenvolvimento e outras informações importantes sobre o projeto. Todo o código e documentações utilizadas para o desenvolvimento do projeto foram hospedados de maneira open source no GitHub. Isso possibilita que outros pesquisadores e interessados na área, possam contribuir com o projeto, realizem melhorias e até mesmo o utilizarem como base para projetos de outras áreas. O acesso ao repositório é pelo link https:/github.com/geraldo7junior/caatinga-digital. O projeto ainda disponibiliza para a comunidade uma API web com os dados sobre a caatinga utilizados e criados a partir da plataforma. Estes dados podem ser consumidos por sistemas de terceiros, serem usados em outras pesquisas e para o fim educacional de conhecimento da região.

A metodologia do Toolkit HCD utilizada mostrou-se eficiente e promissora para pesquisas desta natureza. Foi desenvolvida uma solução que atende as necessidades de diferentes públicos, obtendo feedbacks positivos, pois as funcionalidades da aplicação estão de acordo com o que foi desenhado pelos seus usuários finais. Com a conclusão da primeira versão do Caatinga Digital, foi notado que os testes realizados estão satisfazendo as necessidades dos usuários, com relatos de que a aplicação está ajudando em aulas e pesquisas. Quanto ao público especialista, os testes concentram-se em ajustar funcionalidades e expansão do banco de dados. Ao fim desta fase a aplicação será liberada para o público em geral através das lojas de aplicativos e via web.

\section{Conclusões e Trabalhos futuros}

Era fundamental para a equipe que o público alvo estivesse envolvido em todas as etapas do projeto, garantindo que a solução final realmente fosse útil, para isso, utilizou- 
se a metodologia proposta pelo Toolkit HCD. O Caatinga Digital é um é um projeto multiplataforma que visa unir jogos educativos, internet das coisas e data science para auxílio na divulgação, educação e preservação do ecossistema da Caatinga. Através dos módulos desenvolvidos os usuários podem ter diversão enquanto aprende, podem denunciar crimes ambientes, acompanhar dados e informações do IBGE e do MMA, informações podem ser coletadas dos dispositivos dos usuários para fins de pesquisa, entre outras funcionalidades. Com isso, distintos aspectos ecológicos, educativos e de pesquisa são abordados e apresentados de forma simples para os usuários.

Este trabalho apresenta contribuições para dois grupos distintos. O primeiro é formado por pesquisadores, membros de ONGs, estudantes, professores e entusiastas sobre a caatinga, que agora possuem uma ferramenta que fornece uma série de funcionalidades que são úteis para eles no seu dia-a-dia. O segundo grupo são pesquisadores e desenvolvedores que podem utilizar as APIs do Caatinga Digital para desenvolverem outras aplicações relacionadas a caatinga, ou que podem ir ao repositório do projeto e adaptá-lo para a utilização dos seus módulos para outros cenários e biomas. O uso da metodologia do Toolkit HCD para o desenvolvimento de soluções deste tipo se mostrou promissória e fica como contribuição para a comunidade uma forma de aplicá-la no cenário de ferramentas para a educação ambiental. Além disso, até o presente momento não existem soluções semelhantes desenvolvidas.

Portanto, conforme as análises realizadas e os feedbacks coletados, constata-se que o projeto Caatinga Digital teve uma relevante aceitação pelo seu público alvo e mostra ser uma possível plataforma para a resolução de problemas deste gênero. Esperase que a transmissão do conhecimento através da ferramenta mostre a importância da Caatinga para a sociedade e que todos os módulos unidos e outros podem vir a ser desenvolvidos possam contribuir na preservação deste ecossistema tão rico e valioso.

Como trabalhos futuros espera-se a curto prazo disponibilizar o sistema via web, na Google Play e na App Store. O projeto será continuado ouvindo novas necessidades dos usuários, assim adicionando novas funcionalidades e jogos. Foi percebido um déficit de soluções tecnológicas para esta área na literatura, logo outras sugestões que venham contribuir para a divulgação e preservação deste bioma são bem-vindas.

\section{Referências}

An, D. Y., da Silva, C. D., Ribeiro, D. M. G., da Rocha, P. B. R., Maltinti, C., Nunes, V. B., \& Fávero, R. (2013). Digita-um jogo educativo de apoio ao processo de alfabetização infantil. In Brazilian Symposium on Computers in Education (Simpósio Brasileiro de Informática na Educação-SBIE) (Vol. 24, No. 1, p. 154).

Brasil. (2017) Ministério do Meio Ambiente. Biomas: Caatinga. Disponível em: $<$ http://www.mma.gov.br/biomas/caatinga >. Acesso em: junho de 2017.

Dondi, C., \& Moretti, M. (2007). A methodological proposal for learning games selection and quality assessment. British Journal of Educational Technology, 38, 502.

Drumond, M. A., et al. (2000) "Estratégias para o uso sustentável da biodiversidade da caatinga." Estratégias para o uso sustentável da biodiversidade da caatinga. Petrolina: EMBRAPA/CPTSA. 
VI Congresso Brasileiro de Informática na Educação (CBIE 2017)

Anais do XXVIII Simpósio Brasileiro de Informática na Educação (SBIE 2017)

Ferreira, B. M., Rivero, L., Lopes, A., Marques, A. B., \& Conte, T. (2014). UsabiliCity: um jogo de apoio ao ensino de propriedades de usabilidade de software através de analogias. In Brazilian Symposium on Computers in Education (Simpósio Brasileiro de Informática na Educação-SBIE) (Vol. 25, No. 1, p. 1273).

IDEO. (2014). Human Centered Design Toolkit. Tradução de Tennyson Pinheiro, José Colucci Júnior e Isabela de Melo. 2 ed. San Francisco, California/USA.

Luciano, E. M., Testa, M. G., \& Rohde, L. R. (2007). Gestão de serviços de tecnologia da informação: identificando a percepção de benefícios e dificuldades para a sua adoção. Encontro da Anpad (Enanpad), 31.

Martins, C., Angelo, R. B., \& de Oliveira, H. T. (2013) Potencialidades e limitações de jogos educativos sobre biodiversidade para a construção de práticas de educação ambiental. VII EPEA - Encontro Pesquisa em Educação Ambiental.

Nielsen, J., \& Budiu, R. (2013). Mobile usability. MITP-Verlags GmbH \& Co. KG.

Panosso, M. G., Souza, S. R. D., \& Haydu, V. B. (2015). Características atribuídas a jogos educativos: uma interpretação analítico-comportamental. Revista Psicologia Escolar e Edcuacional, 19(2), 233-242.

Pratas, A. (2014). Creating flat design websites. Packt Publishing Ltd.

Rocha, L. A. G., de Mendonça Cruz, F., \& Leão, A. L. (2015). Aplicativo para educação ambiental. Periódico Eletrônico Fórum Ambiental da Alta Paulista, 11(4).

Siqueira, A. A., Santos Filho, J. V. D., \& Siqueira Filho, J. A. D. (2012). Carolus: a new system for handling electronic botanical collections. Rodriguésia, 63(3), 715-732. 